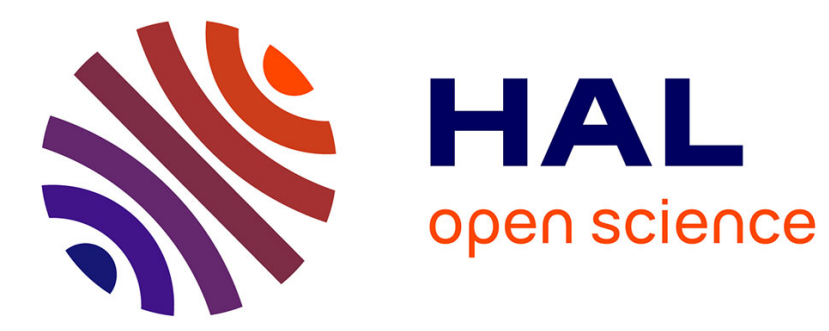

\title{
Pursuit-Evasion Games and Zero-sum Two-person Differential Games
}

\author{
Pierre Bernhard
}

\section{To cite this version:}

Pierre Bernhard. Pursuit-Evasion Games and Zero-sum Two-person Differential Games. Encyclopaedia of Systems and Control, Springer-Verlag, pp.8, 2015, 10.1007/978-1-4471-5102-9_270-1 . hal01215556

\section{HAL Id: hal-01215556 \\ https://hal.inria.fr/hal-01215556}

Submitted on 14 Oct 2015

HAL is a multi-disciplinary open access archive for the deposit and dissemination of scientific research documents, whether they are published or not. The documents may come from teaching and research institutions in France or abroad, or from public or private research centers.
L'archive ouverte pluridisciplinaire HAL, est destinée au dépôt et à la diffusion de documents scientifiques de niveau recherche, publiés ou non, émanant des établissements d'enseignement et de recherche français ou étrangers, des laboratoires publics ou privés.

\section{(ㄷ)(1) $\Theta$}

Distributed under a Creative Commons Attribution - NoDerivatives| 4.0 International 


\title{
Pursuit-Evasion Games and Zero-sum Two-person Differential Games
}

\author{
Pierre Bernhard ${ }^{1}$ \\ June 15, 2013
}

\begin{abstract}
Differential games arose from the investigation, by Rufus Isaacs in the 50's, of pursuit-evasion problems. In these problems, closed-loop strategies are of the essence, although defining what is exactly meant by this phrase, and what is the Value of a differential game, is difficult. For closed-loop strategies, there is no such thing as a "two-sided Maximum Principle", and one must resort to the analysis of Isaacs' equation, a Hamilton Jacobi equation. The concept of viscosity solutions of Hamilton-Jacobi equations has helped solve several of these issues.
\end{abstract}

\section{Historical perspective}

The history of Differential Games (DG in short) starts with Rufus Isaacs, who coined the phrase in his pioneering work of the early '50's [16], which was largely ignored until the publication of his book [15]. Through the investigation of particular problems, Isaacs invented by himself (with his own names) the concepts of state and control variables, of feedback, his "tenet of transition" — better known as Bellman's optimality principle—, the (Hamilton-Jacobi-Caratheodory-)Isaacs equation, barriers, some difficult corner conditions ("equivocal lines"), singular arcs ("universal lines"), etc.

Another very early work was Kelendzerize's chapter "A Pursuit Problem " in the historical book by Pontryagin et al [23], but it lacked closed-loop strategies.

John Breakwell and a few followers $[6,5]$ picked up Isaacs' work where he had left it, still working on particular problems, but adding the power of the computer to analyze the solution of Isaacs' equation via the structure and singularities of fields of extremal trajectories, while most of the literature concentrated on making precise the concepts of closed-loop strategies and of Value of the game. Prominent figures in that quest are N. Krasovskii and A.I. Subbotin [17], W. Fleming [13], A. Friedman [14], A. Blaquière, F. Gérard and G. Leitmann [4], Eliott and Kalton [11] and E. Roxin [26] and P. Varaiya [27] who together invented the concept of non-anticipative strategies.

The major later innovation was Crandal and Lions' viscosity solutions of PDE's $[18,10]$ applied to DG's and its Isaacs equation by Evans and Souganidis [12, 19].

We also refer the reader to the article [25] of another Springer Encyclopaedia.

\section{General set-up}

We shall be interested in (continuous time) two-person zero-sum DG's with complete information, this last phrase meaning that both players know exactly and instantly the state of the system, but (usually) not their opponent's control.

${ }^{1}$ INRIA-Sophia Antipolis Méditerranée, France 
The available space of a short article does not allow us to attempt to give the most general set-up of a zero-sum two-person perfect-information Differential Game. We shall therefore concentrate on a typical class, with a finite dimensional state space, as follows. The data are:

1. A two-player dynamical system with state $x \in \mathbb{R}^{n}$, control variables $u \in \mathrm{U} \subset \mathbb{R}^{\ell}$, $v \in \mathrm{V} \subset \mathbb{R}^{m}$ ( $\mathrm{U}$ and $\mathrm{V}$ will often be assumed compact) and its dynamics

$$
\dot{x}=f(t, x, u, v), \quad x\left(t_{0}\right)=x_{0} .
$$

Denoting $\mathcal{U}$ and $\mathcal{V}$ the sets of measurable functions from $\mathbb{R}$ to $U$ and $V$ respectively, one assumes regularity and growth conditions on $f$ to guarantee existence and uniqueness of the solution $x(\cdot)$ for all $\left(t_{0}, x_{0}\right)$, and all $(u(\cdot), v(\cdot)) \in \mathcal{U} \times \mathcal{V}$.

2. A termination condition, often given by a target set $\mathcal{T} \in \mathbb{R} \times \mathbb{R}^{n}$, open or closed according to necessity, defining a final time as $t_{1}=\inf \{t \mid(t, x(t)) \in \mathcal{T}\}$. If $\mathcal{T}=\{T\} \times \mathbb{R}^{n}$, final time is fixed and equal to $T$. The question of whether there is a finite $t_{1}$ is one of central interest in pursuit-evasion games.

3. Sets of admissible closed-loop strategies $\Phi$ and $\Psi$. One should choose them in such a way that, replacing $(u, v)$ by a pair $(\phi, \psi) \in \Phi \times \Psi$ in the dynamics always produces a (unique) admissible pair of control functions $(u(\cdot), v(\cdot))=\Gamma\left(t_{0}, x_{0} ; \phi, \psi\right) \in \mathcal{U} \times \mathcal{V}$.

4. A performance measure, or pay-off, typically

$$
J\left(t_{0}, x_{0} ; u(\cdot), v(\cdot)\right)= \begin{cases}K\left(t_{1}, x\left(t_{1}\right)\right)+\int_{t_{0}}^{t_{1}} L(t, x(t), u(t), v(t)) \mathrm{d} t & \text { if } t_{1}<\infty \\ \infty & \text { if } t_{1}=\infty\end{cases}
$$

We let

$$
G\left(t_{0}, x_{0} ; \phi, \psi\right):=J\left(t_{0}, x_{0} ; \Gamma\left(t_{0}, x_{0} ; \phi, \psi\right)\right) .
$$

5. A concept of "solution", where the first player wants to minimize the performance index while the second one wishes to maximize it. (In our choice of definition of $J$, we have assumed that player one wants over anything else to make the game terminate. If we define $J$ as the integral even for infinite end-time, Isaacs' tenet of transition may not hold).

If

$$
\inf _{\phi \in \Phi} \sup _{\psi \in \Psi} G\left(t_{0}, x_{0} ; \phi, \psi\right)=\sup _{\psi \in \Psi} \inf _{\phi \in \Phi} G\left(t_{0}, x_{0} ; \phi, \psi\right)=V\left(t_{0}, x_{0}\right),
$$

then $V$ is called the Value function of the game. Several concepts of upper Value and lower Value may be defined (including the first and second terms above) that have to coincide for a Value to exist.

Isaacs' condition In the framework of this short article, we shall always assume that the game satisfies Isaacs' condition. It bears on the hamiltonian $H(t, x, p, u, v):=$ $L(t, x, u, v)+\langle p, f(t, x, u, v)\rangle$ and reads

$$
\forall(t, x, p) \in \mathbb{R} \times \mathbb{R}^{n} \times \mathbb{R}^{n}, \quad \inf _{u \in \mathrm{U}} \sup _{v \in \mathrm{V}} H(t, x, p, u, v)=\sup _{v \in \mathrm{V}} \inf _{u \in \mathrm{U}} H(t, x, p, u, v) .
$$




\section{Strategies and Value}

In pursuit-evasion games, the concept of closed-loop strategies is of the essence, and it is extremely important for all DG's. Yet, allowing state feedback strategies such as $u(t)=\phi(t, x(t)), v(t)=\psi(t, x(t))$, poses a difficult problem : what classes $\Phi$ and $\Psi$ of functions $\phi$ and $\psi$ to allow? The notations $\inf _{\phi}$ or $\sup _{\psi}$ have no meaning if one does not answer that question. Experience tells us that discontinuous feedbacks are necessary to find the solution of many examples, but then existence, or uniqueness, of the solution of the dynamical equation cannot be guaranteed.

Isaacs' K-strategies were a partial attempt to address this issue. More developed concepts were proposed, from limit of piecewise constant, or piecewise open-loop, controls (Fleming [13], Friedmann [14]) to extensions of the notion of solution of a differential equation (Krasovskii, [17]), also proving the existence of a Value. The equivalence of all these Values was an issue until the advent of viscosity solutions of Isaacs' equation.

A tool used to accommodate state-feedback strategies [3] is

Lemma 1 (Berkovitz) If $\mathcal{V} \subset \Psi$, then, $\forall \phi$ for which this expression is well defined,

$$
\sup _{\psi \in \Psi} G\left(t_{0}, x_{0} ; \phi, \psi\right)=\sup _{v(\cdot) \in \mathcal{V}} G\left(x_{0}, t_{0}, \phi, v(\cdot)\right) \text {. }
$$

As a consequence, a saddle-point $\left(\phi^{\star}, \psi^{\star}\right)$ solution is defined by

$$
\forall u(\cdot) \in \mathcal{U}, \forall v(\cdot) \in \mathcal{V} \quad G\left(t_{0}, x_{0} ; \phi^{\star}, v(\cdot)\right) \leq V\left(t_{0}, x_{0}\right) \leq G\left(t_{0}, x_{0} ; u(\cdot), \psi^{\star}\right),
$$

confronting the closed-loop saddle point strategies to open-loop controls only. (This proves useful in the analysis of Nash equilibria of nonzero-sum DG's.)

Another consequence of Berkovitz' lemma is that if a DG has a saddle point in open-loop controls, it is a saddle point over closed-loop controls as well. (But the existence condition may be less stringent for the later.) The relationship between different forms of the strategies has been further clarified by Başar [1, 2].

As far as the existence of the Value is concerned, the problem for a large class of DG's is solved with non-anticipative strategies defined as $\Phi: \mathcal{V} \rightarrow \mathcal{U}$ such that

$$
\forall t,\left[\forall s<t \quad v_{1}(s)=v_{2}(s)\right] \Rightarrow\left[\phi\left(v_{1}(\cdot)\right)(t)=\phi\left(v_{2}(\cdot)\right)(t)\right],
$$

and likewise for $\Psi$, (notice that for this concept of strategies, (2) is the natural formulation of a saddle point), and with the notion of viscosity solution of Isaacs' equation. See theorem 1 below.

\section{Games of pursuit evasion}

An important class of DG's are games of pursuit-evasion. Typically, in these games the state $x$ is composed of a sub-vector $y$ of Pursuer state( $s)$ and a sub-vector $z$ of Evader state $(s)$. The dynamical function $f$ is separated likewise, the dynamics of the Pursuer depending on the Pursuer's control(s) and that of the Evader on the Evader's control( $s$. Typically, the pay-off is time until capture defined as $(t, x(t)) \in \mathcal{T}$ (the target is often called capture set). This form of DG automatically satisfies Isaacs' condition (1). 


\subsection{Qualitative game}

In pursuit-evasion games, the main issue is to distinguish initial states, called capturable, for which a Pursuer's strategy causing finite-time capture against any defense exists, from those, called safe, for which the Evader has a strategy guaranteeing escape against any defense. This is the topic of the qualitative game or game of kind (Isaacs). A theorem of the alternative is one which states that for a particular (class of) game(s), every initial state is either capturable or safe. Such theorems have been proved for classes of Pursuit-Evasion games covering essentially all cases of interest, under Isaacs condition (1) with $L=0$. (Krasovskii [17], Cardaliaguet et al. [7, 9].)

Capturable states are separated from safe states by a barrier, a piecewise smooth manifold which has to be semi-permeable. This means that for all $(t, x)$ on the barrier where this barrier is a smooth manifold with normal $\nu(t, x)$, it should hold that

$$
\min _{u \in \mathrm{U}} \max _{v \in \mathrm{V}}\langle\nu(t, x), f(t, x, u, v)\rangle=\max _{v \in \mathrm{V}} \min _{u \in \mathrm{U}}\langle\nu(t, x), f(t, x, u, v)\rangle=0 .
$$

A minimax pair $(u, v)=(\hat{\varphi}(t, x, \nu), \hat{\psi}(t, x, \nu))$ is called a pair of semipermeable strategies. If the boundary of the capture set is a smooth manifold with local outward normal $n(t, x)$, its usable part is the region where $\inf _{u \in \mathrm{U}} \sup _{v \in \mathrm{V}}\langle n(t, x), f(t, x, u, v)\rangle$ $<0$. The natural barrier is a semi-permeable manifold constructed backward from its boundary (the BUP), with $n$ as final $\nu$ and using the characteristic equations:

$$
\dot{x}=f(x, \hat{\phi}, \hat{\psi}), \quad \dot{\nu}^{t}=-\nu^{t} \frac{\partial f(t, x, \hat{\varphi}, \hat{\psi})}{\partial x} .
$$

(These trajectories are abnormal trajectories of the calculus of variations.) In most examples, only part of the manifold thus constructed is a barrier, and the complete barrier is made of manifolds pieced together according to a junction condition insuring that the corners "do not leak" (Breakwell), analogous to the corner conditions of the next section.

\subsection{Quantitative game}

The quantitative game, or game of degree (Isaacs), is played inside the capture zone, typically with time of capture as the pay-off. It is ruled by Isaacs' equation in a fashion similar to that of games of finite duration (see below). Yet, the interplay between the qualitative and the quantitative game may be quite subtle, and plays a prominent role in determining the actual capture zone. The Value function is usually discontinuous across other barriers inside the capture zone..

\subsection{Other approaches}

Other approaches have been developed to solve games of pursuit-evasion.

An early approach by Pontryagin [22], extended by Pshenichnyi [24], used geometric methods for linear pursuit-evasion games with convex compact controls sets. Krasovskii's stable bridges [17] are a concept close to Isaacs' semi-permeability. Patsko 
and Turova [21] have developed, for some families of DG's, an efficient numerical procedure to compute recursively hypersurfaces of constant time-to-capture, whose discontinuities display the barriers. Cardaliaguet, Quincampoix and Saint-Pierre [8] have developed a theoretical and numerical procedure building on Aubin's viability theory, which requires less regularity on the data than other approaches.

Provided that care be applied, a quantitative game may be transformed into a family of qualitative games - an approach used by Krasovskii, Blaquière et al. and Cardaliaguet et al.- , and conversely, a fruitful approach is to investigate capturability of initial states as a function of a parameter defining the "size" of the capture set, imbedding the qualitative game into a quantitative game of the type game of approach.

\section{Games of finite duration}

Wherever termination of the game is not an issue, the major tool in investigating a DG is Isaacs' equation, a partial differential equation bearing on the Value function:

$$
\begin{aligned}
& \forall(t, x) \notin \mathcal{T}, \quad \frac{\partial V}{\partial t}(t, x)+\min _{u \in \mathrm{U}} \max _{v \in \mathrm{V}} H\left(t, x, \nabla_{x} V, u, v\right)=0, \\
& \forall(t, x) \in \mathcal{T}, \quad V(t, x)=K(t, x) .
\end{aligned}
$$

For any DG where all trajectories are transverse to the boundary $\partial \mathcal{T}$, and with adequate regularity conditions on the data (and still under condition 1), it holds that

Theorem 1 The DG has a Value in nonanticipative strategies, which is the only bounded, uniformly continuous viscosity solution of the equation obtained by changing signs in (3) as $-\partial V / \partial t-\min _{u \in U} \max _{v \in V} H=0$. And all other Values coincide.

One possible way to solve Isaacs' equation is via the investigation of its field of characteristics. Their equations are Isaacs' retrograde path equations : let $(\hat{u}, \hat{v})=$ $(\hat{\varphi}(t, x, p), \hat{\psi}(t, x, p))$ be the saddle point of $H(t, x, p, u, v)$, assumed here to be unique, one integrates from the target set backward

$$
\begin{aligned}
\dot{x} & =f(t, x, \hat{u}, \hat{v}), \\
\dot{p} & =-\left(\frac{\partial H(t, x, p, \hat{u}, \hat{v})}{\partial x}\right)^{t} .
\end{aligned}
$$

The above equations are similar to Pontryagin's maximum principle equations. However, a major difference lies in the corner conditions. While Pontryagin's theorem extends to control theory the Erdman-Weierstrass condition stating that the adjoint vector (here $p$ ) is continuous along an extremal trajectory, in (4)-(5), $p$ is to coincide with $\nabla_{x} V$, and may be discontinuous along an extremal trajectory. These discontinuities cannot be found by a local analysis along an isolated trajectory, and require that a complete field of extremals be constructed, synthesizing a state feedback strategy.

The analysis of the conditions that hold at such corners : equivocal manifolds (Isaacs), envelope manifolds (Breakwell), focal manifolds (Merz), has been a large part of the early, Isaacs-Breakwell, theory. It has been for its larger part synthesized by 
Bernhard [3], except a general constructive analysis of focal manifolds which had to wait until [20].

The absence of a "two-sided Pontryagin principle" for closed-loop Differential Games forces one to resort to the solution of Isaacs' equation, or an equivalent. This is the reason why no practical method of solution exists beyond a state dimension of 3 or 4 , counting time if the game is not time-invariant. An exception is the linear quadratic game. (See that article.)

\section{Conclusion}

Except for very particular games, "solving" a DG remains a difficult task. Numerical methods suffer the famous "curse of dimensionality". Moreover, many of them strive to compute the Value function. But the optimal strategies typically depend on the gradient of the Value function, requiring a stronger convergence of the approximation algorithms than pointwise, or $C^{0}$ or $L^{2}$, if they are to be computed as well. Further advances in numerical algorithms tackling this problem would be useful, as well as uncovering new classes of DGs for which further analytical results could be obtained.

\section{References}

[1] Tamer Başar. Informationally nonunique equilibrium solutions in differential games. SIAM Journal on Control and Optimization, 15:636-660, 1977.

[2] Tamer Başar and Geert-Jan Olsder. Dynamic Noncooperative Game Theory. Academic Press, 1982.

[3] Pierre Bernhard. Singular surfaces in differential games, an introduction. In Peter Haggedorn, GeertJan Olsder, and Hans Knobloch, editors, Differential games and Applications, volume 3 of Lecture Notes in Information and Control Sciences, pages 1-33. Springer Verlag, Berlin, 1977.

[4] Austin Blaquière, Franọise Gérard, and George Leitmann. Quantitatve and Qualitative Games. Academic Press, New York, 1969.

[5] J. V. Breakwell and A. V. Merz. Toward a complete solution of the homicidal chauffeur game. In Yu-Chi Ho and George Leitmann, editors, Proceedings of the First International Conference on the Theory and Applications of Differential Games, Amherst, 1969.

[6] John V. Breakwell. Lecture notes. In P. Hagedorn, H. W. Knobloch, and G-J. Olsder, editors, Theory and Applications of Differential Games, number 3 in Springer Lecture Notes in Control and Information Sciences, pages 70-95. Springer Verlag, Berlin, 1977.

[7] Pierre Cardaliaguet. A differential game with two players and one target. SIAM journal on Control and Optimization, 34:1441-1460, 1996. 
[8] Pierre Cardaliaguet, Marc Quincampoix, and Patrick Saint-Pierre. Set-valued numerical methods for optimal control and differential games. In A. Nowak, editor, Stochastic and Differential Games. Theory and Numerical Methods, Annals of the International Society of Dynamic Games, pages 177-247. Birkhaüser, 1999.

[9] Pierre Cardaliaguet, Marc Quincampoix, and Patrick Saint-Pierre. Pursuit differential games with state constraints. SIAM Journal on Control and Optimization, 39:1615-1632, 2001.

[10] M. G. Crandal and P-L. Lions. Viscosity solutions of Hamilton Jacobi equations. Transactions of the American Mathematical Society, 277:1-42, 1983.

[11] Robert J. Elliot and Nigel J. Kalton. The existence of value in differential games of pursuit and evasion. Journal of Differential Equations, 12:504-523, 1972.

[12] L. C. Evans and P. E. Souganidis. Differential games and representation formulas for solutions of Hamilton-Jacobi-Isaacs equations. Indiana University Mathematical Journal, 33:773-797, 1984.

[13] Wendel K. Fleming. The convergence problem for differential games. Mathematical Analysis and Applications, 3:102-116, 1961.

[14] A. Friedman. Differential Games. Wiley, 1971.

[15] Rufus Isaacs. Differential Games, a Mathematical Theory with Applications to Optimization, Control and Warfare. John Wiley and Sons, New York, 1965.

[16] Rufus P. Isaacs. Games of pursuit. Technical Report P-257, The Rand corporation, 1951.

[17] N. Krasovskii and A. Subbotin. Jeux Différentiels. MIR, Moscow, 1977.

[18] P-L. Lions. Generalized Solutions of Hamilton-Jacobi Equations. Pitman, 1982.

[19] P-L. Lions and P. E. Souganidis. Differential games, optimal control, and directional derivatives of viscosity solutions of Bellman's and Isaacs' equations. SIAM journal on Control and optimization, 23:566-583, 1985.

[20] Arik Melikyan and Pierre Bernhard. Geometry of optimal trajectories around a focal singular surface in differential games. Applied Mathematics and Optimization, 52:23-37, 2005.

[21] Valery S. Patsko and Varvara L. Turova. Level sets of the value function in differential games with the homicidal chauffeur dynamics. International Game Theory Review, 3:67-112, 2001.

[22] L. S. Pontryagin. Linear differential games I and II. Soviet Math Doklady, 8:769771 and 910-912, 1967.

[23] L. S. Pontryagin, V. G. Boltyanskii, R. V. Gamkrelidze, and E. F. Mishenko. The Mathematical Theory of Optimal Processes. John Wiley \& sons, New York, 1962. 
[24] B. N. Pshenichnyi. Linear differential games. Automation and Remote Control, 29:55-67, 1968.

[25] Marc Quincampoix. Differential games. In N. Meyers, editor, Encyclopaedia of Complexity and System Science, pages 1948-1956. Springer, 2009.

[26] Emilio O. Roxin. The axiomatic approach in differential games. Journal of Optimization Theory and Applications, pages 153-163, 1969.

[27] P. Varaiya and Y. Lin. Existence of saddlepoint in differential games. SIAM Journal on Control, 7:141-157, 1969. 